\title{
CNS-targeted gene therapy improves survival and motor function in a mouse model of spinal muscular atrophy
}

\author{
Marco A. Passini, Jie Bu, Eric M. Roskelley, Amy M. Richards, S. Pablo Sardi, \\ Catherine R. O'Riordan, Katherine W. Klinger, Lamya S. Shihabuddin, and Seng H. Cheng \\ Genzyme Corporation, Framingham, Massachusetts.
}

\begin{abstract}
Spinal muscular atrophy (SMA) is a neuromuscular disease caused by a deficiency of survival motor neuron (SMN) due to mutations in the SMN1 gene. In this study, an adeno-associated virus (AAV) vector expressing human SMN (AAV8-hSMN) was injected at birth into the CNS of mice modeling SMA. Western blot analysis showed that these injections resulted in widespread expression of SMN throughout the spinal cord, and this translated into robust improvement in skeletal muscle physiology, including increased myofiber size and improved neuromuscular junction architecture. Treated mice also displayed substantial improvements on behavioral tests of muscle strength, coordination, and locomotion, indicating that the neuromuscular junction was functional. Treatment with AAV8-hSMN increased the median life span of mice with SMA-like disease to 50 days compared with 15 days for untreated controls. Moreover, injecting mice with SMA-like disease with a human SMN-expressing self-complementary AAV vector - a vector that leads to earlier onset of gene expression compared with standard AAV vectors - led to improved efficacy of gene therapy, including a substantial extension in median survival to 157 days. These data indicate that CNS-directed, AAV-mediated SMN augmentation is highly efficacious in addressing both neuronal and muscular pathologies in a severe mouse model of SMA.
\end{abstract}

\section{Introduction}

Spinal muscular atrophy (SMA) is an autosomal recessive neuromuscular disorder caused by mutations in the survival motor neuron 1 (SMN1) gene and loss of encoded SMN protein (1). The lack of SMN results in motor neuron degeneration in the ventral (anterior) horn of the spinal cord, which leads to weakness of the proximal muscles responsible for crawling, walking, neck control, and swallowing and the involuntary muscles that control breathing and coughing (2). Consequently, SMA patients present with increased tendencies for pneumonia and other pulmonary problems such as restrictive lung disease $(3,4)$. The onset of disease and degree of severity are determined in part by the phenotypic modifier gene $S M N 2$, which is capable of making a small amount of $\operatorname{SMN}(5,6)$. Thus, patients with a high SMN2 copy number (3 to 4 copies) exhibit a less severe form of the disease (referred to as types II or III), whereas 1 to 2 copies of SMN2 typically result in the more severe type I disease $(7,8)$. Currently, there are no effective therapies for SMA.

A fundamental strategy for treating this monogenic disorder is to increase SMN levels in SMA patients. This could potentially be accomplished by 2 distinct approaches. One approach is to utilize gene therapy vectors to provide a continuous source of exogenous SMN. A second approach is to modulate the endogenous SMN2 gene with small molecules that activate the SMN2 promoter (9-16) or correct the SMN2 pre-mRNA splicing pattern $(10,17-22)$. The alteration of SMN2 splicing also can be realized with antisense oligonucleotides and transsplicing RNAs (23-27). However, while modulating SMN2 in vitro increased SMN levels and reconstituted nuclear gems in SMA

Conflict of interest: All authors are paid employees of Genzyme Corporation. Citation for this article: J Clin Invest. 2010;120(4):1253-1264. doi:10.1172/JCI41615 cell lines, efficacy studies with small molecule drugs have not translated to measurable improvements in the clinic (28).

Several animal models of SMA have been generated $(2,29)$. The best characterized of these is a murine model that incorporated the entire human SMN2 locus onto an SMN-null background (30). The aggressive disease severity in this SMA mouse model $\left(\mathrm{SMN}^{-/-}, \mathrm{hSMN} 2^{+/+}, \mathrm{SMN} \Delta 7^{+/+}\right)$recapitulates many of the aberrant phenotypes observed in the SMA type I patients $(2,30)$. Phenotypes in this mouse model include motor neuron cell loss, skeletal muscle atrophy, aberrant neuromuscular junctions (NMJ), behavioral deficits, paralysis, and a shortened life span of about 2 weeks (30). It is not known whether long-term survival can be achieved in this animal model, as testing of several therapeutic drugs to date has shown only a modest improvement in median survival $(16,31-33)$.

In the current study, we tested whether direct injections of a recombinant adeno-associated virus (AAV) vector encoding hSMN into the CNS is efficacious in this severe SMA mouse model $\left(\mathrm{SMN}^{-/-}, \mathrm{hSMN2} 2^{+/+}\right.$, SMN $\left.\Delta 7^{+/+}\right)$. Our data showed that many of the hallmark pathologies associated with SMA were corrected by gene therapy, and a single administration of the $A A V$ vector without the aid of nutritional support increased median survival to 50 days. Remarkably, median survival was increased to 157 days when injections were performed with a self-complementary AAV (scAAV) vector, which is a recombinant virus defined as having a double-stranded DNA genome that results in earlier onset of gene expression compared with regular single-stranded AAV (34). The information in this study demonstrates that this severe SMA mouse model can be rescued by somatic gene transfer to the CNS and supports the development of AAV-based technologies as a potential therapeutic approach for SMA. 

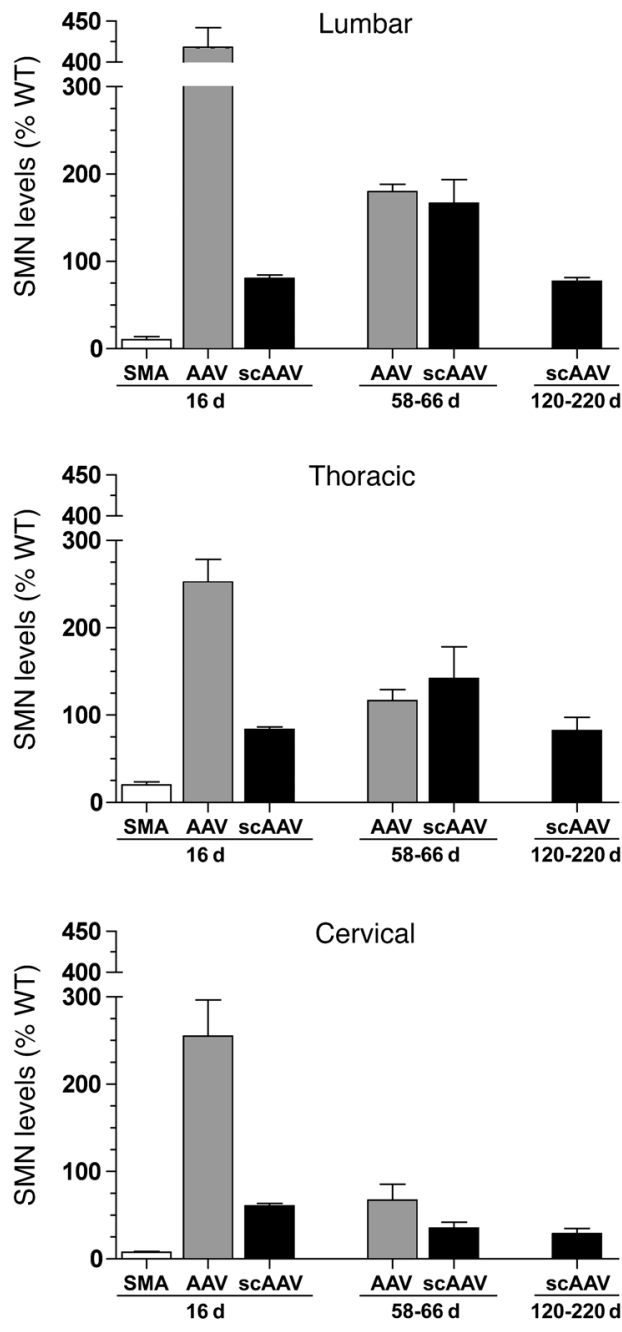

\section{Results}

CNS delivery of AAV8-hSMN resulted in bigh levels of expression in spinal cord. Newborn (P0) SMA mice were injected with an AAV8-serotype vector containing the human SMN1 cDNA (AAV8-hSMN) into both cerebral lateral ventricles and into the upper lumbar spinal cord for a total dose of 5.0 e10 genome copies per pup. i.c.v. injections alone did not contribute to appreciable AAV transduction patterns in the brain (data not shown) but nevertheless generated substantial targeting of the cervical spinal cord that was not achievable with lumbar-only injections (Supplemental Figure 1; supplemental material available online with this article; doi:10.1172/JCI41615DS1). Thus, the combination of i.c.v. and lumbar injections in P0 mice provided broad, widespread transduction of the spinal cord.

Treated and control mice were randomly assigned into either a survival cohort in which all the mice were left undisturbed until they reached a humane end point or into a cohort in which all the mice were killed at 16 days for age-matched comparisons with end-stage untreated SMA mice. Examination of the 16-day-old mice injected with AAV8-hSMN showed elevated SMN levels throughout the spinal cord. AAV8-hSMN treatment resulted in a 34.0- and 3.6-fold increase in SMN levels in the lumbar segment compared with untreated SMA and untreated WT mice,

\section{Figure 1}

Treatment with gene therapy increased SMN levels in the spinal cord. Western blots were performed on the lumbar, thoracic, and cervical segments at 16, 58-66, and 120-220 days after injection. The Western blots from the 3 segments were quantified and, to control for protein levels, SMN was normalized to $\beta$-tubulin and plotted as a percentage of age-matched WT. SMA, untreated knockout ( $n=5$ at 16 days); AAV, AAV8-hSMN-treated SMA mice ( $n=7$ at 16 days; $n=5$ at 58-66 days); scAAV, scAAV8-hSMN-treated SMA mice ( $n=5$ at each time point). Data represent the mean \pm SEM.

respectively (Figure 1). This increase in SMN levels extended into other segments of the spinal cord, including a greater than 2-fold increase above WT in the thoracic and cervical segments. AAV8hSMN-treated SMA mice that survived to 58-66 days also contained elevated levels of SMN, although the values were less than those observed at 16 days (Figure 1).

Human SMN was expressed in neurites and in a subset of motor neurons. Immunohistochemical and in situ hybridization analysis of spinal cord tissue sections at 16 days showed AAV8-derived hSMN expression in the dorsal and ventral horns (Figure 2, A-D). hSMN expression was detected in both neurons and glia. Closer examination of the transduced cells showed hSMN was present in a punctate pattern throughout the cytosol and in gem-like structures in the nucleus that are implicative of functional SMN (Figure 2E). Furthermore, hSMN was also detected in neurites in distinct granule-like structures that spanned the length of the dendrites and axons (Figure 2, F-H). These observations suggest that hSMN was located in the appropriate intracellular compartments of neurons that may benefit from its reconstitution. AAV8-hSMN-treated SMA mice also showed colocalization of hSMN and mouse choline acetyltransferase (mChAT), demonstrating that the viral vector transduced a subset of spinal cord motor neurons (Figure 2, I-K). Associated with this observation was a modest preservation in the number of motor neurons in treated SMA mice, although the values were only significantly increased in the thoracic segment (Figure 2L). However in all cases, the number of motor neurons in AAV8-treated SMA mice was significantly less than that observed in age-matched WT animals (Figure 2L).

CNS-directed gene therapy improved the size of myofibers in skeletal muscle. The impact of CNS-directed gene therapy on the physiology of the skeletal muscle was examined. The quadriceps (proximal), gastrocnemius (distal), and intercostal (respiratory) muscles were selected for analysis, as they display marked degeneration in SMA disease. Examination of the myofibers from all 3 muscle groups of 16-day-old WT mice showed cells with varying cross-sectional areas ranging between 100 and $500 \mu \mathrm{m}^{2}$ (Figure 3A) and with a mean of approximately $260 \mu \mathrm{m}^{2}$ (Figure 3B). With increasing age (58-66 days), the sizes of the myofibers from WT mice were larger, exhibiting a mean cross-sectional area of more than $550 \mu \mathrm{m}^{2}$. In contrast, the myofibers of untreated SMA mice at 16 days were small, with the majority of cells exhibiting a cross-sectional area of less than $100 \mu \mathrm{m}^{2}$ (Figure 3A). Analysis of the AAV8-hSMNtreated SMA mice showed a reduction in the percentage of myofibers that exhibited a cross-sectional area of less than $100 \mu \mathrm{m}^{2}$ at 16 days $(P<0.001$; Figure $3 \mathrm{~A})$. On average, the myofibers were smaller than those in WT mice but were approximately 2-fold larger than those of untreated SMA mice ( $P<0.001$; Figure 3B). The sizes of the myofibers of AAV-treated SMA mice were larger at 58-66 days, indicating that the muscle was growing with age 

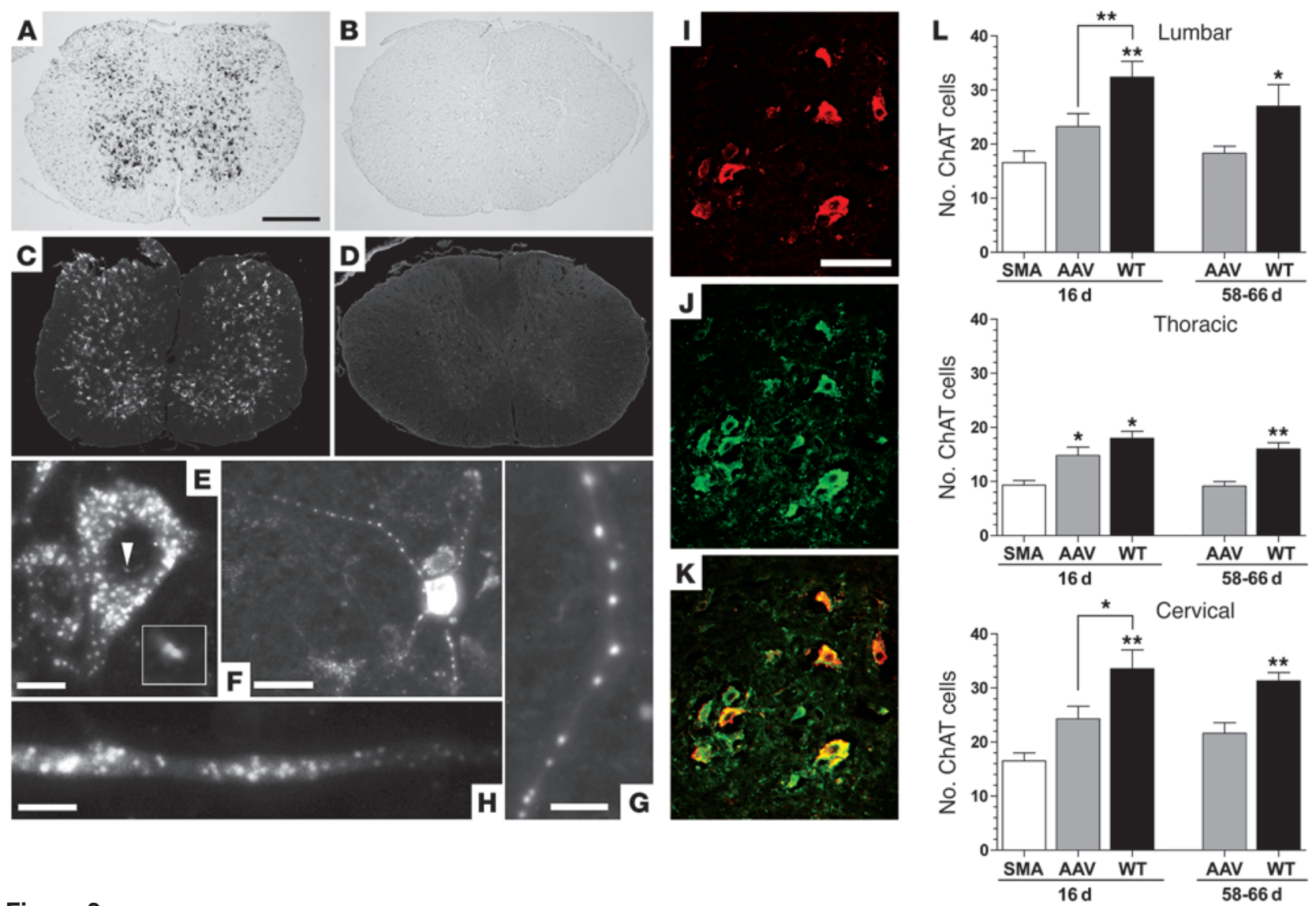

\section{Figure 2}

Human SMN is expressed in the proper intracellular compartments and in motor neurons with AAV8-hSMN treatment. In situ hybridization (A and $\mathbf{B})$ and immunohistochemistry $(\mathbf{C}-\mathbf{K})$ of the lumbar segment in AAV8-hSMN-treated (A, C, E-K) and untreated (B and $\mathbf{D})$ SMA mice at 16 days $(\mathbf{A}-\mathbf{D})$ and 58-66 days (E-K) after injection. The low level of endogenous hSMN in untreated SMA mice was below the threshold of detection using these assays (B and $\mathbf{D}$ ). Vector-derived hSMN was abundantly detected in the cytoplasm and in the nucleus of transduced cells, as illustrated by the pair of gem-like structures in the nucleus (E, the arrowhead points to the hSMN immunosignal magnified in the inset). hSMN was also detected in the dendrites $(\mathbf{F}$ and $\mathbf{G})$ and in the axons $(\mathbf{H})$ of neurons. The colocalization of hSMN (I) with mChAT (J) showed that a subset of transduced cells consisted of motor neurons (K). Shown are the average numbers of ChAT immunopositive cell counts from the lumbar, thoracic, and cervical segments at both time points (L). SMA ( $n=8$ at 16 days); AAV ( $n=8$ at 16 days, $n=5$ at $58-66$ days); WT, untreated WT ( $n=8$ at 16 days, $n=5$ at 58-66 days). Values represent the mean \pm SEM. Scale bars: 500 microns (A-D); 10 microns (E and $\mathbf{H})$; 50 microns (F); 12 microns (G), 100 microns (I-K). Statistical comparisons were performed with 1-way ANOVA and Bonferroni's multiple post hoc tests at 16 days and with unpaired 2-tailed Student's $t$ tests at $58-66$ days $(\mathbf{L}) .{ }^{*} P<0.05 ;{ }^{* \star} P<0.01$.

(Figure 3B). The average myofiber cross-sectional areas at 58-66 days were $67 \%, 76 \%$, and $82 \%$ that of WT mice in the quadriceps, gastrocnemius, and intercostal, respectively (Figure 3B). Hence, reconstitution of hSMN in a subset of the motor neurons was partially effective at rescuing skeletal muscle physiology.

Treatment with AAV8-hSMN improved the architecture of the NMJ. Analysis of the NMJ from untreated SMA mice at 16 days showed abnormal accumulation of neurofilament protein at the presynaptic termini (Figure 4A). Approximately $75 \%-90 \%$ of the presynaptic termini from the quadriceps, gastrocnemius, and intercostal of the untreated SMA animals displayed this hallmark pathology of SMA disease (Figure 4F). In contrast, the majority of the presynaptic termini from the AAV8-hSMN-treated SMA mice did not contain this aberrant structure (Figure 4, B and D). Only 10\%-25\% of the presynaptic termini from the AAV-treated SMA mice at day 16 and $5 \%$ of those from days 58-66 showed this pathology $(P<0.001$; Figure 4F). AAV8-hSMN-treated SMA mice exhibited more branching at the presynaptic termini when compared with WT animals
(Figure 4, B-E). On the postsynaptic NMJ, $\alpha$-bungarotoxin staining revealed a proper "pretzel-like" structure indicative of a functional network of acetylcholine receptors in the treated mice (Figure 4, $\mathrm{B}-\mathrm{E})$. These results are congruent with the earlier observation of improved muscle physiology in the AAV-treated SMA mice.

Treatment with AAV8-hSMN restored motor function in SMA mice. Mice were subjected to periodic behavioral testing using assays that had previously been validated for this animal model $(35,36)$. Treated SMA mice at 16 days were healthier than their untreated age-matched counterparts, who looked emaciated and were often paralyzed (Figure 5A). The AAV8-hSMN-treated mice gained more weight than the SMA controls. However, they remained significantly smaller than the WT animals (Figure 5B). In the righting reflex test that measures overall muscle strength and coordination, AAV8treated SMA mice showed a significant improvement starting at day 8 that was normalized by days $12-16(P<0.001$; Figure 5C). In contrast, the latency of untreated SMA mice to right from a supine position was severely impaired, especially by day 16 (Figure 5C). 
A
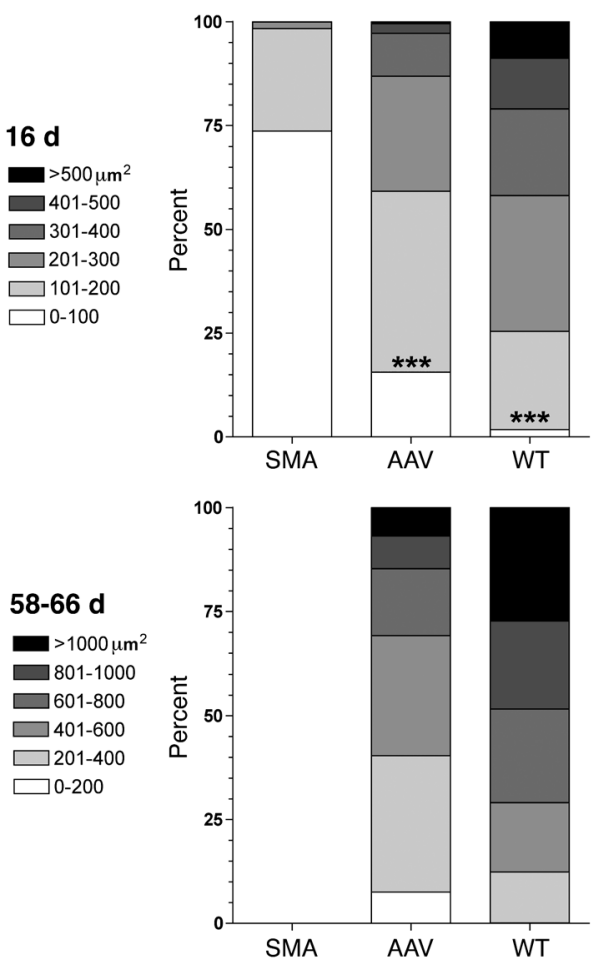

Gastrocnemius
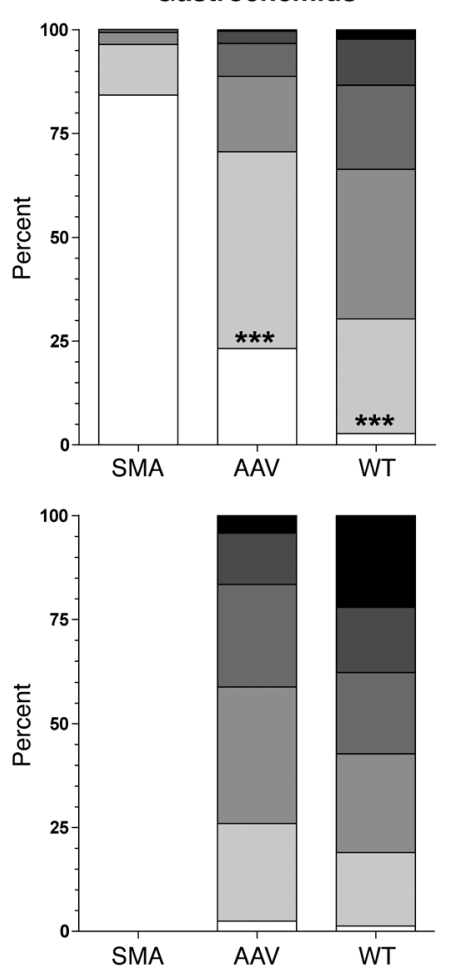

Intercostal
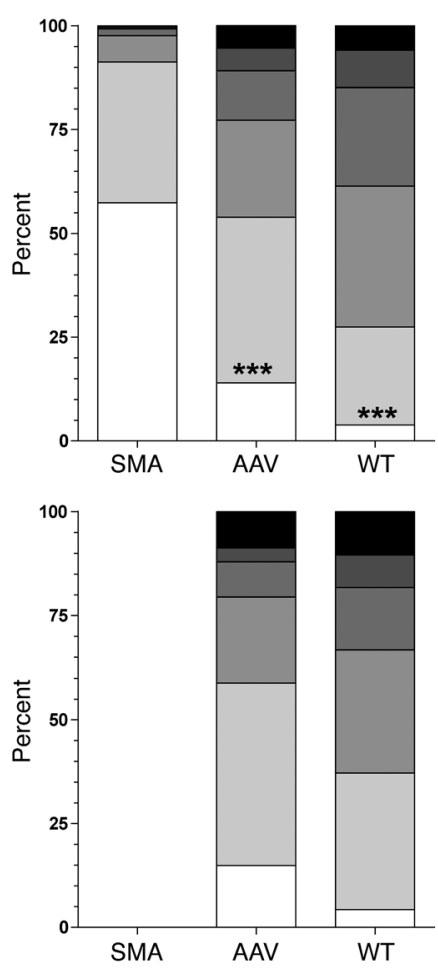

Intercostal
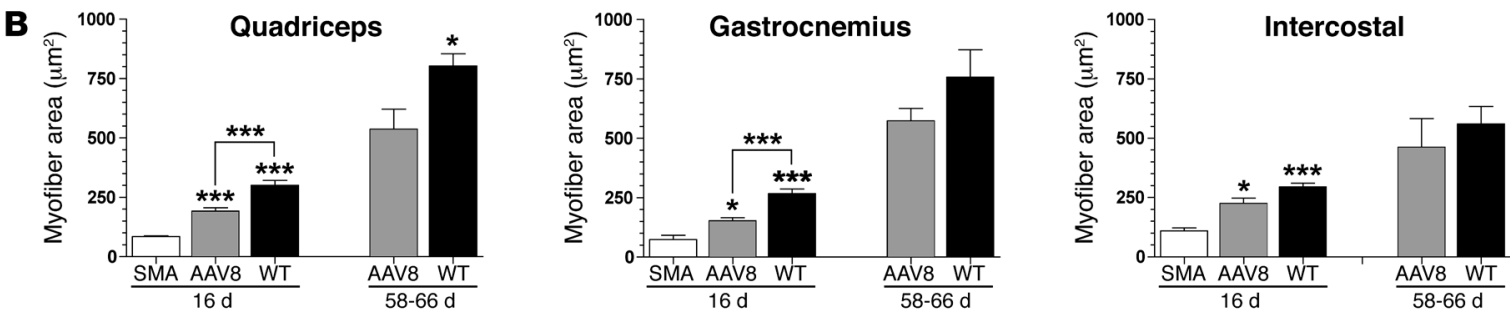

Figure 3

The myofiber cross-section area was increased with AAV8-hSMN treatment. Stacked graphs of the quadriceps, gastrocnemius, and intercostal muscles from 16 days (first row) and 58-66 days (second row) showed that the relative distribution of myofiber sizes was similar between the AAV8-hSMN-treated SMA mice and the untreated WT controls (A). Furthermore, the number of myofibers with a cross-section area of less than $100 \mu \mathrm{m}^{2}$ was significantly reduced with AAV8-hSMN treatment (A). The overall mean of the myofiber cross-section area was higher with treatment compared with untreated SMA at 16 days (B). In addition, at 58-66 days, the average myofiber size in treated SMA mice approached WT levels, particularly in the gastrocnemius and intercostal muscles (B). SMA ( $n=8$ at 16 days); AAV ( $n=8$ at 16 days; $n=5$ at $58-66$ days); WT ( $n=8$ at 16 days; $n=5$ at 58-66 days). Values represent the mean \pm SEM. Statistical comparisons were performed with 1 -way ANOVA and Bonferroni's multiple post hoc tests at 16 days (A and $\mathbf{B}$ ) and with unpaired 2-tailed Student's $t$ tests at $58-66$ days (B). ${ }^{*} P<0.05 ;{ }^{* \star \star} P<0.001$.

Grip strength was used as a direct measurement of muscle strength in the forelimbs and hind limbs. AAV8-treated SMA mice performed substantially better than untreated SMA mice in the grip-strength test, but less well than WT mice $(P<0.001$; Figure 5D). The pattern of improvement in the grip strength corresponded with the partial recovery of the average myofiber size in the gastrocnemius and quadriceps (Figure 3B). Animals were also subjected to a hind-limb splay test as a qualitative measure of muscle coordination. AAV-treated SMA mice exhibited a score that was intermediate between untreated SMA and WT mice $(P<0.001$; Figure 5E). A fourth behavior test employed was the negative-geotaxis test, which measures spatial locomotive behavior (Figure 5F). This assessment involved placing the mice to face downward on an incline and measuring their natural tendency to reorient them- selves to face the top of the incline. AAV-treated mice showed a significant improvement in their spatial locomotive function, performing at a level similar to WT animals $(P<0.001$; Figure 5F).

Gene therapy significantly increased survival in SMA mice. SMA mice treated with AAV8-hSMN showed a significant increase in median life span to 50 days, compared with 17, 15, and 15 days for AAV8null-treated, saline-treated, and untreated SMA mice, respectively $(P<0.0001$; Figure 6A). All the AAV8-hSMN-treated SMA mice were alive at 15 days, and $87.5 \%$ of the mice were viable at 19 days compared with none in untreated SMA controls. Analysis of the AAV8-hSMN-treated mice by Kaplan-Meier showed a bimodal survival curve in which the first group died between 17 and 27 days and the second group between 58 and 66 days (Figure 6A). In the first group, the majority of the treated SMA mice were ambulatory 

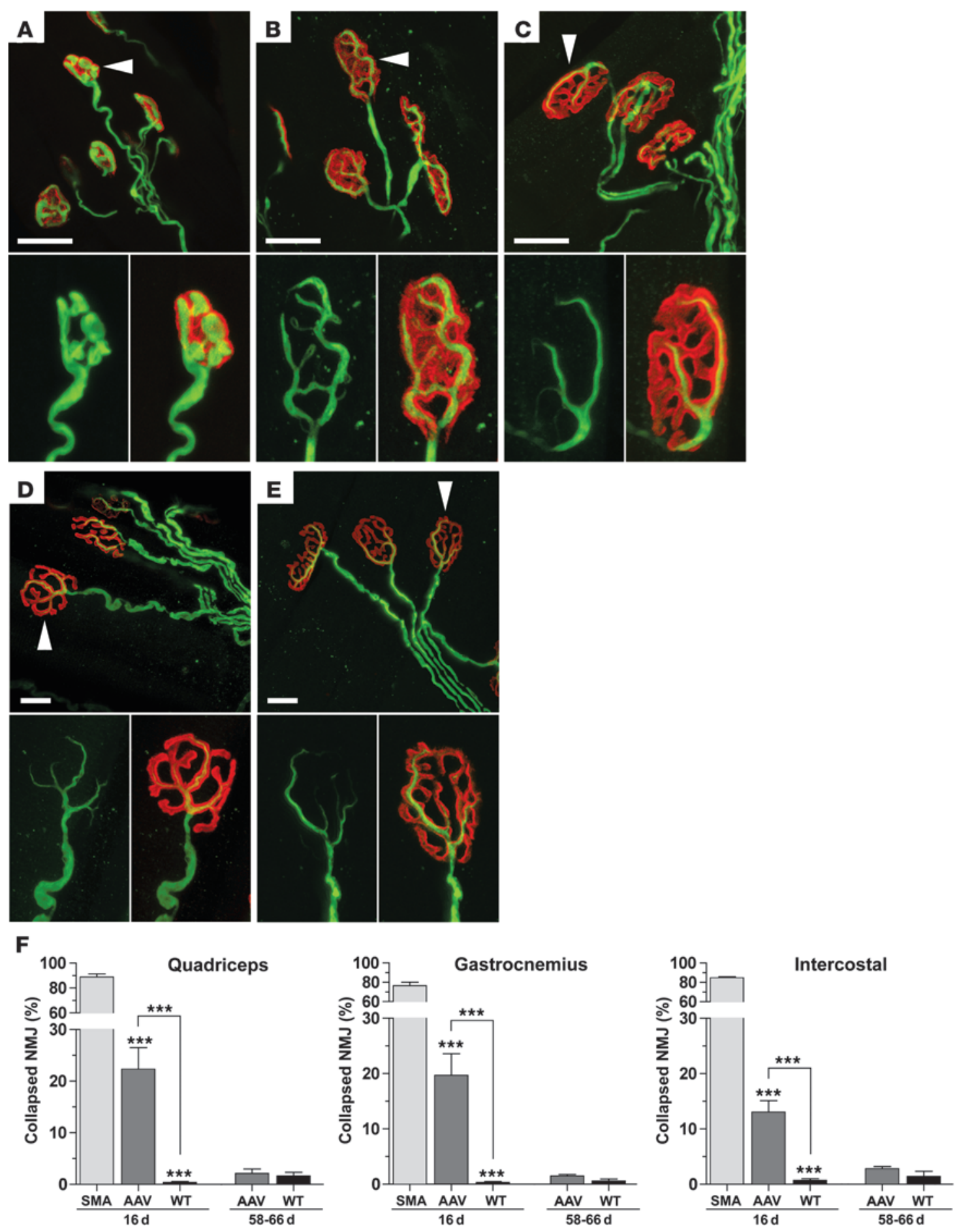

\section{Figure 4}

The NMJ in the quadriceps, gastrocnemius, and intercostal muscles was improved with gene therapy. Shown are the NMJ from the quadriceps of untreated SMA (A), AAV8-hSMN-treated SMA (B and D), and untreated WT (C and E) mice at 16 days (A-C) and at 58-66 days (D and E) after injection. The pre- and postsynaptic NMJ was labeled with a neurofilament antibody (green) and with $\alpha$-bungarotoxin staining (red). The arrowhead in each main panel points to the NMJ that is highlighted in the inset below. The percentage of NMJs that contained a collapsed structure similar to that shown in panel A was determined (F). WT ( $n=8$ at 16 days: $n=5$ at 58-66 days); SMA ( $n=8$ at 16 days) ( $n=8$ at 16 days; $n=5$ at 58-66 days), WT ( $n=8$ at 16 days; $n=5$ at 58-66 days). Data represent the mean \pm SEM. Statistical comparisons were performed using 1-way ANOVA and Bonferroni's multiple post hoc tests for each muscle group at 16 days and with unpaired 2-tailed Student's $t$ tests at $58-66$ days $(\mathbf{F}) .{ }^{* * *} P<0.001$. Scale bars: 20 microns $(\mathbf{A}-\mathbf{E})$. 
A
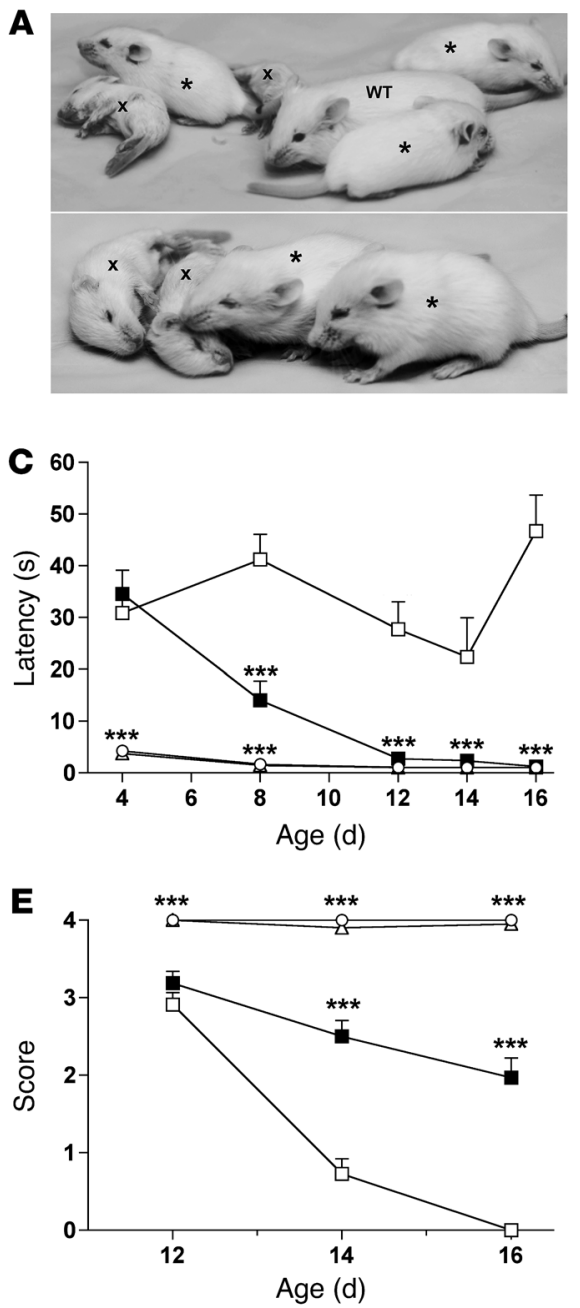
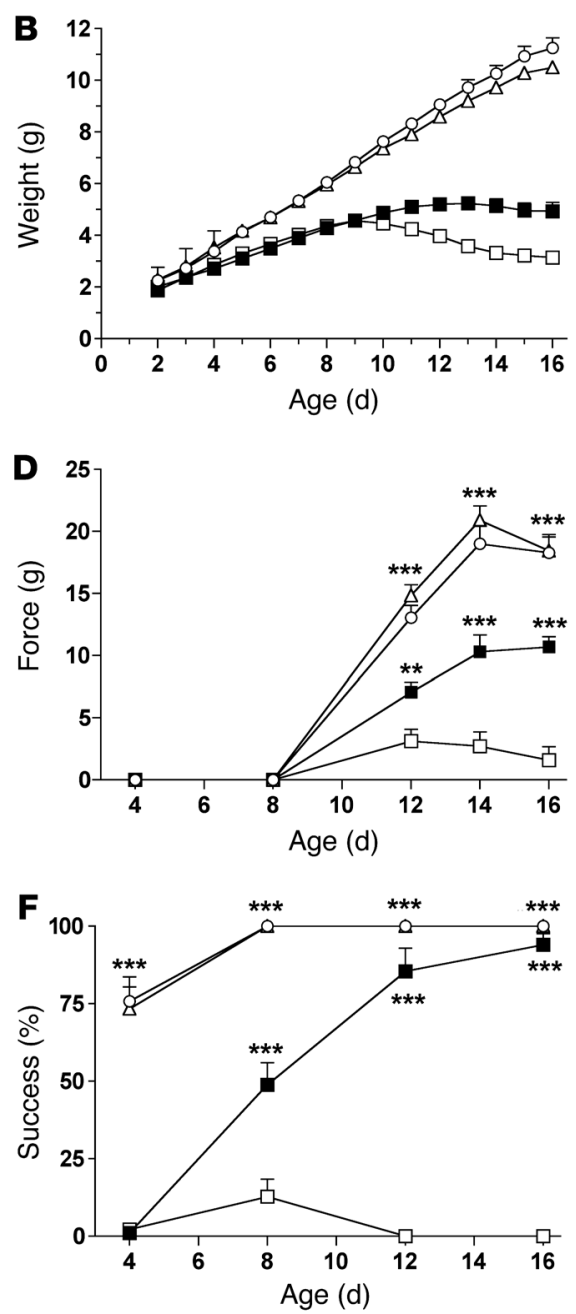

\section{Figure 5}

Treated SMA mice showed significant improvements on behavioral tests. Treated SMA (asterisk) and untreated WT mice were substantially fitter than untreated SMA mice (labeled $\mathrm{x}$ ) at 16 days (A). Treated SMA mice were also significantly heavier than untreated SMA controls from day 11 and onwards (B). Treated SMA mice performed significantly better than untreated SMA mice on the righting reflex (C), grip-strength (D), hind-limb splay (E), and negative-geotaxis (F) tests. Treated SMA mice were statistically similar to WT and heterozygote mice on the righting reflex and negative-geotaxis tests at $12-16$ days (C and $\mathbf{F}$ ). Untreated SMA mice ( $n=11$, open squares); AAV8hSMN-treated SMA mice $(n=16$, closed squares); untreated heterozygote mice ( $n=16$, open triangles); untreated WT mice ( $n=16$, open circles). Data represent the mean \pm SEM. Statistical comparisons were performed for each time point using 1-way ANOVA and Bonferroni's multiple post hoc tests. ${ }^{* \star} P<0.01$; ${ }^{* \star *} P<0.001$.

euthanasia. Rather, $60 \%$ of the scAAV8treated SMA mice were sacrificed due to a sudden appearance of respiratory distress, which included audible clicking gasps when breathing and a decreased rate of respiration. The remaining $40 \%$ of the scAAV8treated SMA mice were sacrificed for other humane reasons, including 1 or more of the following: greater than $20 \%$ loss in body weight, inability to urinate, ulceration of the eye, and chronic dehydration.

but were stunted in growth and were invariably found dead in their cage. The second group at 58-66 days was also ambulatory, and the mice gained weight but subsequently developed severe hind-limb necrosis that required their euthanasia. Hind-limb necrosis was not caused by vector-derived hSMN, as long-term expression with AAV8-hSMN in WT mice did not produce this phenotype (data not shown). Rather, hind-limb necrosis is a secondary phenotype of unknown relevance to SMA disease that typically arises after 4-6 weeks in this mouse model (31).

The relative merits of a self-complementary AAV vector were examined by administering $1.7 \mathrm{e} 10$ genome copies of scAAV8-hSMN into the cerebral lateral ventricles and upper lumbar spinal cords of P0 SMA mice. Despite a 3-fold decrease in dose, treatment with scAAV8hSMN resulted in a striking improvement in median survival of 157 days, which was a $214 \%$ and $881 \%$ increase over AAV8-hSMN-treated and untreated SMA mice, respectively $(P<0.0001$; Figure $6 \mathrm{~B})$. At 66 days, $88 \%$ of the scAAV8-treated SMA mice were still alive as opposed to none in those administered with AAV8-hSMN. Furthermore, $41 \%$ of the scAAV-treated mice showed greater than one logfold (>1000\%) increase in survival. The scAAV8-treated SMA mice exhibited healthy body scores, appeared well groomed, gained weight, and were ambulatory throughout their life (Supplemental Video 1). Interestingly, scAAV8-treated SMA mice developed only a very mild hind-limb necrosis that did not progress to a phenotype requiring
Analysis of scAAV8-hSMN expression and efficacy in SMA mice. To better understand the basis for the observed increase in survival with scAAV8-hSMN, additional SMA mice were treated at P0, sacrificed at 16 or 64 (58-66 d) days after injection, and analyzed with the long-lived scAAV8-treated mice from the survival curve (Figure 6B). At 16 days, SMN expression levels from the scAAV8-hSMN group were approximately $60 \%-90 \%$ of those observed in WT animals (Figure 1). These levels were substantially less than those achieved with AAV8-hSMN treatment at this time point. In the scAAV8hSMN-treated SMA mice, SMN levels in both the lumbar and thoracic segments were above or at WT levels at 58-66 and 120-220 days, respectively (Figure 1). In contrast, SMN levels in the cervical spinal cord remained relatively low at all time points.

Differences in spinal cord transduction patterns were also observed with the 2 viral vectors. In contrast to AAV8-hSMN, histological analysis of scAAV8-hSMN-treated SMA mice showed hSMN expression was largely restricted to neurons (Figure 7). Furthermore, double immunostaining with hSMN and mChAT showed a significant increase in the percentage of motor neurons transduced with scAAV8-hSMN compared with AAV8-hSMN (Figure $8 \mathrm{~A}$ ). The more efficient targeting of motor neurons with scAAV correlated with a significant increase in the number of ChATpositive cells (Figure 8, B-D). Analysis of the NMJ in the quadriceps and intercostal muscles at 16 days also showed a significant 

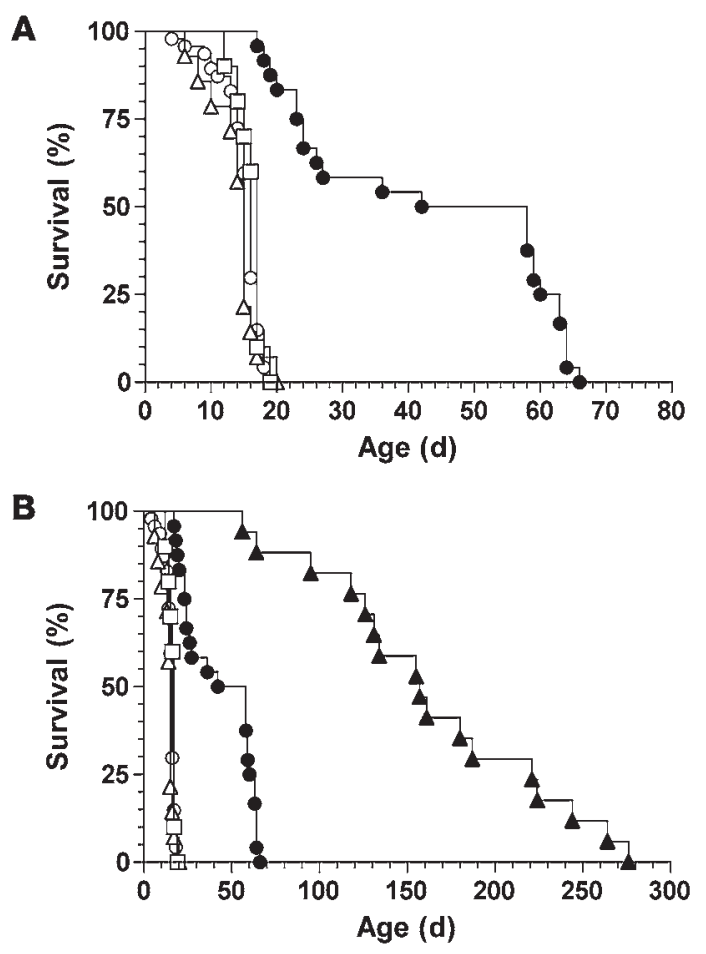

decrease in the number of collapsed structures with scAAV-hSMN compared with AAV8-hSMN (Figure 8, E and F). However, there was an increase in the number of aberrant NMJs at 216-269 days that was concomitant with the decline of motor neuron cell counts in the scAAV-hSMN group (Figure 8, B-F).

\section{Discussion}

Our approach to treating this monogenic disorder was to administer AAV vectors encoding hSMN directly into the CNS. This study was performed in a severe mouse model that recapitulated many of the aberrant phenotypes observed in SMA type 1 patients $(2,30)$. Our results demonstrated that reconstituting SMN levels in the CNS was sufficient to abate the disease manifestations in both the CNS and skeletal muscle. A one-time, CNS-directed therapy treated a wide variety of skeletal muscle groups, including the proximal, distal, and respiratory muscles. SMN levels were increased throughout the spinal cord with treatment, but there were no detectable levels of vector-derived hSMN protein or mRNA in the skeletal muscle from CNS injections (data not shown). The lack of transgene expression in muscle suggests that the improvement in muscle physiology was a direct consequence of CNS treatment. These observations are consistent with SMA transgenic lines showing that CNS treatment was required for efficacy, but increasing SMN levels in the muscle alone had no therapeutic benefit (37). Furthermore, our study showed that somatic gene transfer is highly efficacious when applied after the completion of motor neuron development in severely affected mice, which may translate to efficacy with early postnatal intervention due to the potential plasticity in young children.

AAV8-hSMN treatment produced only a modest preservation in the number of motor neurons in SMA mice. Yet the size of the myofibers and the structure of the NMJs from 3 independent skeletal muscle groups were similar between the WT control mice and

\section{Figure 6}

Gene therapy increased longevity of SMA mice. Untreated SMA mice ( $n=34$, open circles) had a median survival of 15 days (A). SMA mice treated at P0 with either saline ( $n=14$, open triangles) or AAV8-null ( $n=10$, open square) had median survivals of 15 and 17 days, respectively $(P>0.05)$. SMA mice treated at P0 with AAV8-hSMN $(n=24$, closed circles) had a median survival of 50 days $(P<0.0001)$, which was a $233 \%$ increase in longevity compared with untreated SMA mice (A). The survival curve revealed 2 groups of AAV8-hSMN-treated SMA mice, a first group that was found dead by 27 days and a second group that was sacrificed at 58-66 days (A). Treatment with scAAV8hSMN showed an even greater increase in survival (B). SMA mice treated at P0 with scAAV8-hSMN ( $n=17$, closed triangles) had a median life span of 157 days $(P<0.0001)$ compared with 16 days in untreated SMA mice ( $n=47$, open circles) (B). As a reference, the survival plots of the SMA mice treated with AAV8-hSMN, AAV8-null, and saline were also included (B).

the AAV8-hSMN-treated SMA mice. Taken together, these data suggest that reconstituting SMN levels in a subset of motor neurons may compensate for the loss of other neurons by innervating myofibers that had lost their original connection. While there was no direct evidence for collateral sprouting onto new myofibers, the high degree of branching at the axon termini indicates that increasing SMN levels increased the number of neurites. Moreover, transduction of motor neurons with scAAV8 was more efficient than with AAV8, which correlated to an overall increase in the number of motor neurons in the scAAV8-treated group. Thus, the potential of more motor neurons participating in the remolding of the NMJ may help explain the enhanced therapeutic benefit observed with scAAV8-hSMN.

A hallmark pathology in SMA animals and in humans is the abnormal structure of the NMJ. The presynaptic NMJ contains pathological accumulation of neurofilament protein at the axonal termini of motor neurons, and the postsynaptic NMJ contains a plaque-like structure consistent with the abnormal clustering of acetylcholine receptors (38-41). The combination of both irregularities results in a collapsed NMJ, which is characterized by the abnormal communication between the muscle and nerve that ultimately results in deinnervation and muscle atrophy (38-41). CNS-directed gene therapy partially resolved the abnormal architecture of the pre- and postsynaptic NMJ, which also correlated with functional improvements on a battery of behavioral tests. This rescue may have been achieved by hSMN directly modulating the NMJ, such as transporting molecules required for the normalization of the presynaptic termini $(42,43)$. The localization of hSMN in the axon of transduced neurons observed in the current study is consistent with such a role. An alternative explanation is that increasing hSMN may have improved the efficiency of spliceosomes to modify genes responsible for $\mathrm{NMJ}$ function $(43,44)$. Both explanations may not be mutually exclusive, and future studies are required to further elucidate the relative contributions of each mechanism.

Long-term survival in this animal model had not yet been achieved, presumably because of its severe and aggressive natural history. In previous studies, intraperitoneal injections of the histone deacetylase inhibitor trichostatin A showed only a modest increase in median survival to 19 days compared with 16 days in SMA controls (16). This survival benefit was doubled when trichostatin A was combined with nutritional support (31). Intramuscular injections with a recombinant lentiviral vector capable of 


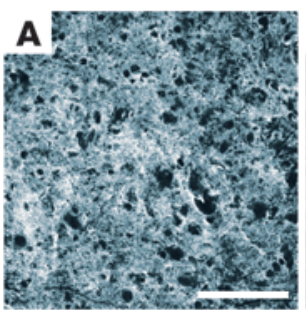

D

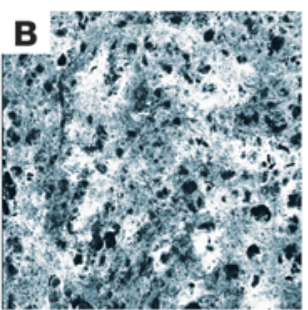

hSMN

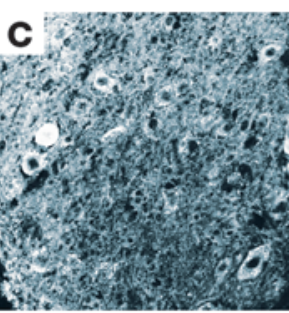

mGFAP
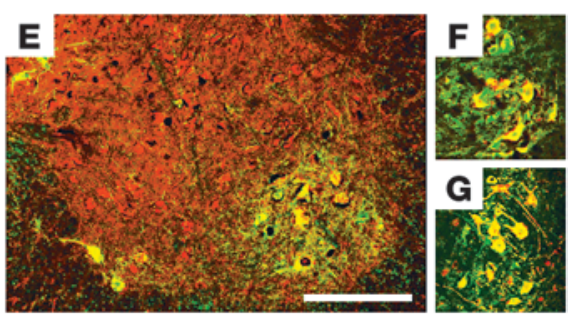

Overlay
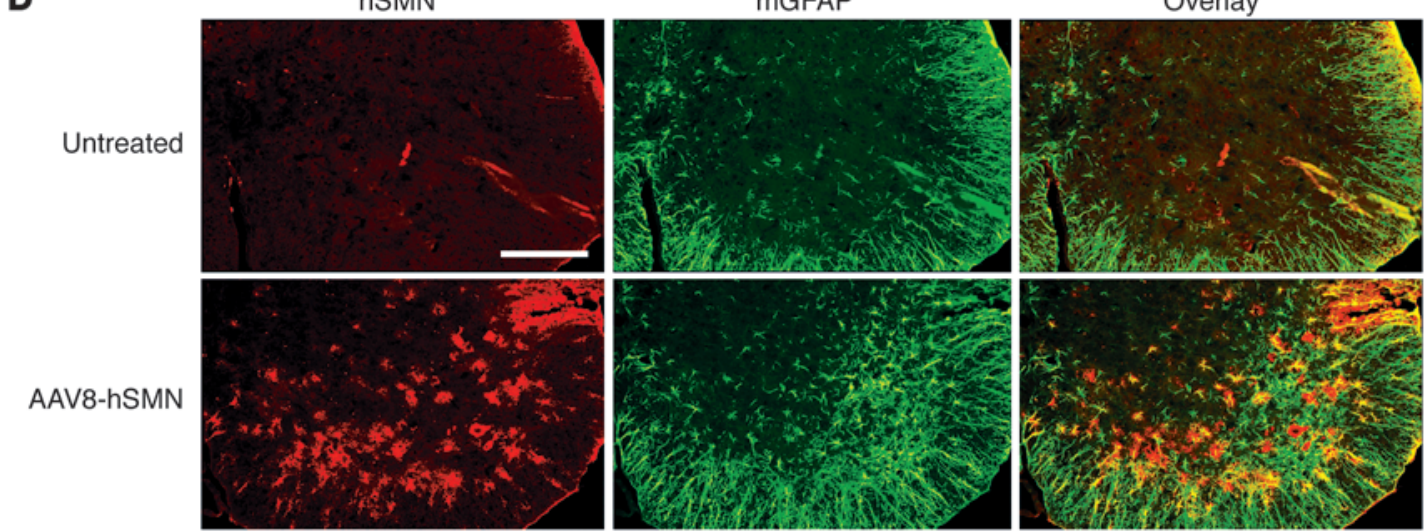

AAV8-hSMN
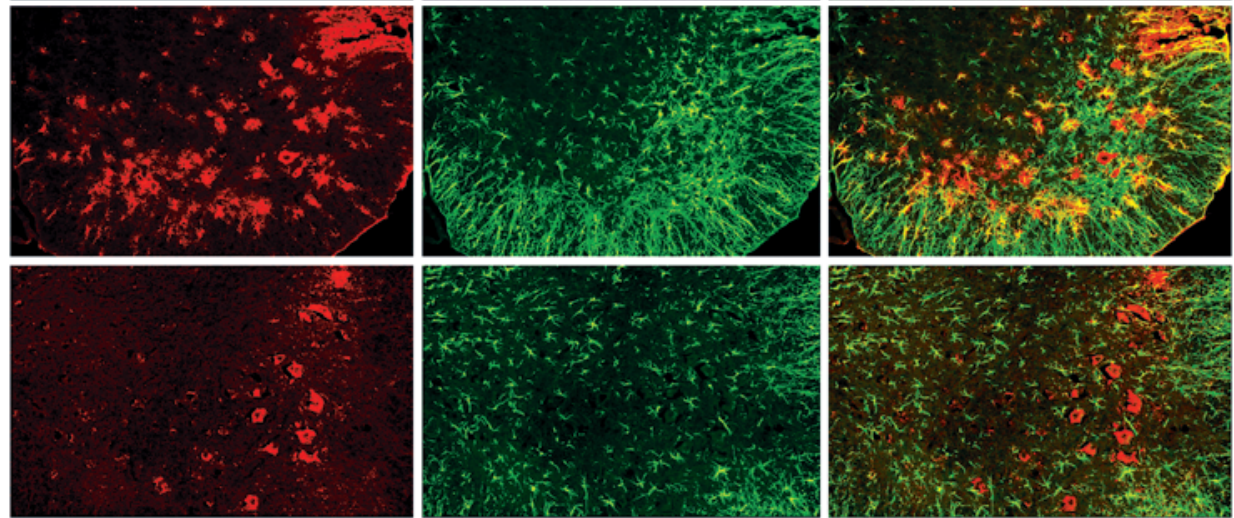

\section{Figure 7}

Comparison of AAV vector tropism in the lumbar spinal cord. Human SMN immunostaining was performed on frozen tissue sections from untreated SMA (A and D), AAV8-hSMN-treated SMA (B, D, and F), and scAAV8-hSMN-treated SMA (C-E, and G) mice at 16 days (A-D, $\mathbf{F}$, and $\mathbf{G}$ ) and 157 days (E) after injection. A diffuse hSMN immunostaining pattern consistent with glial cell morphology was observed at 16 days with AAV8-hSMN (B and $\mathbf{D}$ ). Doubling immunolabeling (yellow) of hSMN (red) and mGFAP (green) confirmed that a subset of the AAV8-hSMN-transduced cells were astrocytes (D). In contrast, scAAV8 treatment resulted in hSMN expression only in distinct cell bodies with neuronal morphology (C), which did not colocalize with GFAP (D). Double immunolabeling (yellow) of hSMN (red) and the motor neuron marker mChAT (green) confirmed that a subset of cells transduced by scAAV8-hSMN (E and G) and AAV8-hSMN (F) were motor neurons. hSMN expression (red) was also observed in the interneuronal cell layers of the spinal cord with both viral vectors, as exemplified by scAAV8-hSMN at 157 days (E). Scale bars: 100 microns (A-C); 200 microns (D and $\mathbf{E}$ ).

retrograde axonal transport and intraspinal injections of neural stem cells each provided an increase in median survival to 18 days $(32,33)$. In contrast, the current study showed increases in median survival of 50 and 157 days for AAV8 and scAAV8 respectively, thus demonstrating that long-term survival is achievable in this animal model. Interestingly, the end-stage respiratory distress observed in many of the scAAV8-hSMN-treated SMA mice (216 to 269 days) correlated with both a decrease in motor neuron counts in the thoracic spinal cord and an increase in abnormal NMJs in the intercostal muscles (Figure 8, C and E). However, there may be additional components of the disease in the viscera, such as the cardiac and gastrointestinal systems $(45,46)$, which may also require treatment to prevent the eventual demise of treated SMA mice.

The viral vectors have different properties in the spinal cord that may account for the enhanced efficacy observed with scAAV8hSMN. The 2-fold increase in the number of motor neurons transduced, combined with the lack of expression in glia cells, suggests there was more efficient uptake of the scAAV8 vector than of the
AAV8 vector in neurons. Thus, targeting of neurons that may benefit from the reconstitution of hSMN was more effectively achieved with scAAV8 compared with AAV8. The differences in CNS tropism may not be simply due to the differences in the promoters regulating hSMN expression, as the 0.4 -kb human $\beta$-glucuronidase (GUSB) promoter in the context of an AAV vector does support expression in all neural cell types including astrocytes (47). Thus, scAAV vectors may have additional intrinsic properties in the CNS despite having the same amino acid capsid sequence as their singlestranded counterpart (AAV). This was demonstrated to be the case for AAV serotype- 9 vectors, in which scAAV9 crossed the bloodbrain barrier significantly better than $\operatorname{AAV} 9(48,49)$. Furthermore, treatment with scAAV8-hSMN in the current study resulted in only mild hind-limb necrosis, suggesting scAAV8 may modulate the peripheral vasculature or blood flow by an unknown mechanism.

It is not known why there was a bimodal survival curve for the AAV8-hSMN-treated group. One possible explanation is that there were 2 populations of SMA mice with differing degrees of 
A
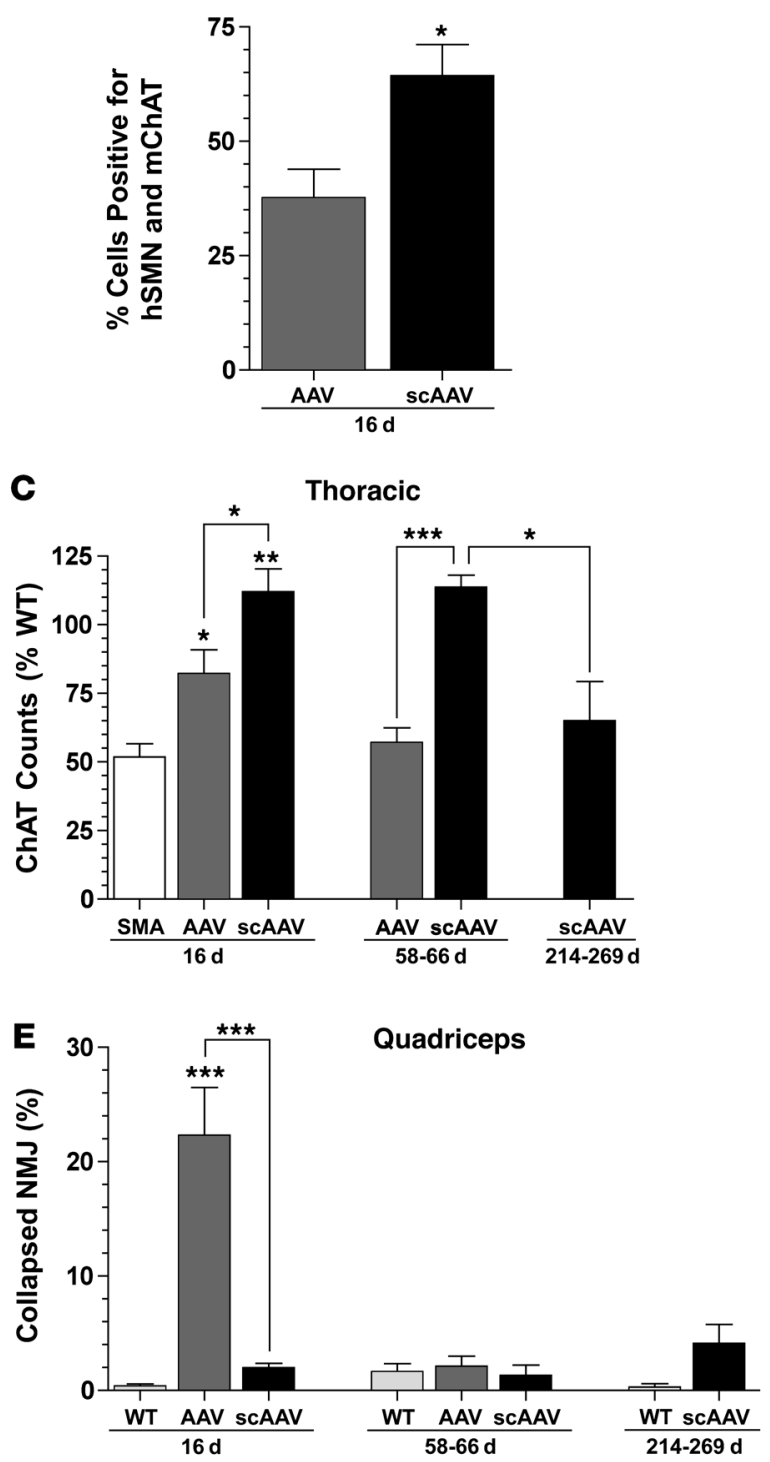

B

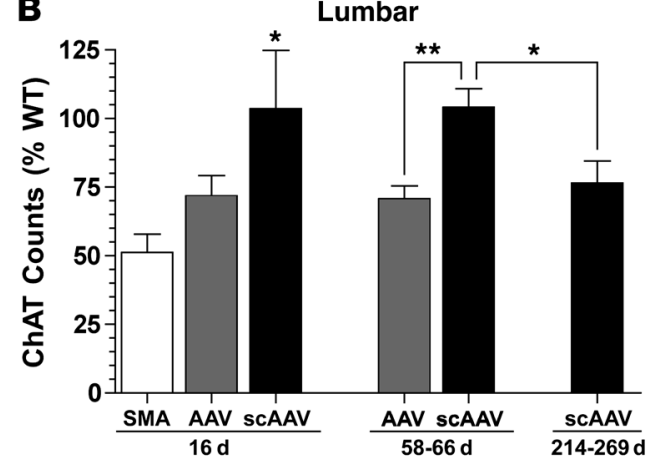

D

Cervical

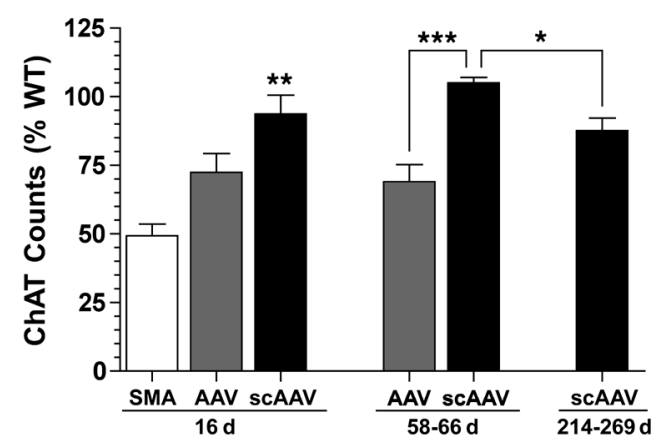

F

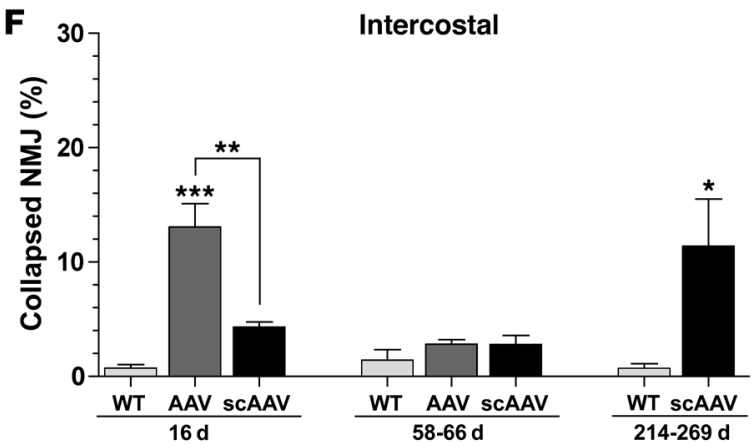

Figure 8

ScAAV8-hSMN expression increased motor neuron counts and improved the NMJ in SMA mice. Shown is the percentage of mChAT immunopositive cells that colocalized with hSMN expression in the thoracic-lumbar region at 16 days after injection (A). Shown are the average numbers of $\mathrm{mChAT}$ immunopositive cells in the lumbar (B), thoracic (C), and cervical (D) segments and the average percentages of collapsed NMJs in the quadriceps (E) and intercostal $(\mathbf{F})$ muscles at 16, 58-66, and 214-269 days. As a reference for panels $\mathbf{E}$ and $\mathbf{F}$, 75\%-90\% of $\mathrm{NMJ}$ in the quadriceps and intercostal muscles of untreated SMA mice contained an aberrant collapsed structure at 16 days (see Figure 4F). SMA ( $n=8$ at 16 days), AAV, AAV8-hSMN ( $n=8$ at 16 days; $n=5$ at 58-66 days); scAAV ( $n=5$ at each time point); WT ( $n=8$ at 16 days; $n=5$ each at 58-66 and 216-269 days). Data represent the mean \pm SEM. Statistical comparisons were performed with 1-way ANOVA and Bonferroni's multiple post hoc tests at 16 days (B-F). The unpaired 2-tailed Student's $t$ tests compared (a) the 2 vectors to each other at 16 days $(\mathbf{A})$ and 58-66 days (B-D); (b) the relative number of ChAT cells in the 58- to 66-day and 214- to 269-day groups with scAAV8-hSMN treatment (B-D); (c) the relative number of abnormal NMJs between the age-matched untreated WT and scAAV8-hSMN-treated SMA mice at 214-269 days (E and $\mathbf{F})$. ${ }^{\star} P<0.05 ;{ }^{* \star} P<0.01 ;{ }^{* \star \star} P<0.001$.

disease severity at the time of intervention. It is plausible that SMA mice with more advanced disease on the day of birth may be less amenable to AAV8-hSMN therapy, which would be consistent with the modest response from the group that died between 17 and 27 days. In this scenario, the improved therapeutic potential of scAAV8-hSMN may impart this vector the ability to rescue a larger spectrum of severely affected mice. Another explanation for the bimodal survival curve may be potential differences in immune responses against the viral capsids in treated SMA mice. Additional studies that investigate the immunological properties of AAV8 in SMA mice will be required to address this issue.

In conclusion, hSMN gene augmentation was highly efficacious in treating the pathological, functional, and survival phenotypes of a severe mouse model of SMA. The impressive increase in survival demonstrated that this animal model could be rescued despite its aggressive natural history. Targeting the CNS alone was suf- 
ficient to address both the neuronal and muscular pathologies, suggesting that additional injections into skeletal muscle may not be required in the clinic. Future studies that investigate spinal cord motor neuron targeting and safety in a large animal, such as the nonhuman primate, will help determine the path forward for this therapeutic modality into the clinic.

\section{Methods}

$A A V$ vectors. The open reading frame of the human SMN1 cDNA (NCBI accession number NM000344) was cloned into a shuttle plasmid containing either the AAV2 inverted terminal repeats (ITR) and the $1.6-\mathrm{kb}$ cytomegalovirus enhancer/chicken $\beta$-actin (CBA) promoter or the scAAV2 ITR and the $0.4-\mathrm{kb}$ GUSB promoter. The size constraint of the recombinant genome in the scAAV packaging reaction required the use of a small promoter (34). Thus, the $0.4-\mathrm{kb}$ GUSB promoter was chosen because it is ubiquitously expressed throughout the CNS $(47,50)$. The recombinant plasmids were each packaged into AAV serotype- 8 capsid by triple-plasmid cotransfection of human 293 cells, and virions were column purified as reported (51). The resulting vectors, AAV8-hSMN (AAV2/8-CBA-hSMN) and scAAV8-hSMN (scAAV2/8-GUSB-hSMN), possessed titers of 8.3 e12 and 2.8 e12 genome copies/ml, respectively. As a control, a third vector was generated in which the hSMN1 cDNA was replaced with a stuffer sequence to generate AAV8-null (AAV2/8-CBA-null) at a titer of 1.0 e13 genome copies per $\mathrm{ml}$.

Animal procedures. All procedures were performed under a protocol approved by the Genzyme Corp. Institutional Animal Care and Use Committee. Heterozygote $\left(\mathrm{SMN}^{+/-}, \mathrm{hSMN2} 2^{+/+}\right.$, and $\left.\mathrm{SMN} \Delta 7^{+/+}\right)$breeding pairs were mated (30). On the day of birth (P0), pups received 3 injections of $2 \mu \mathrm{l}$ each into the cerebral lateral ventricles of both hemispheres and the upper lumbar spinal cord. The AAV-hSMN vectors were injected at full strength, and thus the total doses were $5.0 \mathrm{e} 10$ and $1.7 \mathrm{e} 10$ genome copies for AAV8-hSMN and scAAV8-hSMN, respectively. The AAV8-null vector was diluted with saline to titer match the AAV8-hSMN dose of 5.0 e10 genome copies. All the injections were performed with a finely drawn glass micropipette needle as described (50). Following the injections, the pups were toe clipped and genotyped (30) to identify SMA (SMN-/, hSMN2 ${ }^{+/+}$, $\left.\mathrm{SMN} \Delta 7^{+/+}\right)$, heterozygote, and $\mathrm{WT}\left(\mathrm{SMN}^{+/+}, \mathrm{hSMN} 2^{+/+}, \mathrm{SMN} \Delta 7^{+/+}\right)$mice. All the litters were culled to 7 pups to control for litter size, and some litters were not injected to serve as untreated controls.

Western blots. For biochemical analysis, treated and untreated mice at 16 and 58-66 days were perfused with PBS, and the spinal cords were dissected and separated into the lumbar, thoracic, and cervical segments and then snap-frozen in liquid nitrogen. Tissues were homogenized at a final concentration of $50 \mathrm{mg}$ protein $/ \mathrm{ml}$ using T-Per lysis buffer and protease inhibitor cocktail (Pierce). The homogenates were cleared by centrifugation, and the protein concentration was measured by BCA assay (Pierce). Ten to twenty micrograms of homogenate protein was resolved on a $4 \%-12 \%$ SDS-PAGE, transferred to nitrocellulose membrane, and probed with mouse monoclonal anti-SMN (1:5,000; BD Biosciences) and rabbit polyclonal anti- $\beta$-tubulin (1:750; Santa Cruz Biotechnology Inc.) antibodies. The membranes were incubated with infrared secondary antibodies (1:20,000; LI-COR Biosciences), and protein bands were visualized by quantitative fluorescence using Odyssey software (LI-COR Biosciences). Molecular weight markers confirmed the sizes of the bands.

Immunohistochemistry. For histological analysis, treated and untreated mice were perfused with $4 \%$ paraformaldehyde ( $\mathrm{pH} 7.4$ ); the spinal cords were removed and placed in $30 \%$ sucrose for $48-72$ hours, embedded in OCT, and cut into $10-\mu \mathrm{m}$ frozen sections with a cryostat. Spinal cord sections were blocked for 1 hour at room temperature (RT) and then incubated with either a mouse monoclonal anti-SMN antibody (1:200 dilution;
BD Biosciences) to locate AAV-derived hSMN, a goat polyclonal anticholine acetyl transferase (ChAT) antibody (Millipore; 1:100 dilution) to identify motor neurons, or a rabbit polyclonal anti-glial fibrillary acidic protein (GFAP) antibody (Sigma-Aldrich, 1:2,500 dilution) to detect astrocytes. Primary antibodies were incubated for 1 hour at RT followed by an overnight incubation at $4^{\circ} \mathrm{C}$ in a humidified chamber. Spinal cord sections were then incubated for 1 hour at RT with either a biotinylated antimouse, Cy3-conjugated anti-goat, or FITC-conjugated anti-rabbit secondary antibody (1:250 dilution; Jackson ImmunoResearch). To increase the SMN and ChAT immunopositive signal, a TSA signal amplification kit (PerkinElmer) or a citric acid antigen retrieval protocol (Vector Labs) was performed according to the manufacturers' instructions. Sections were cover-slipped with Vectashield mounting media (Vector Labs). In situ hybridization was performed as described (50) using a nonradioactive digoxigenin-labeled hSMN antisense riboprobe.

Motor neuron counting. The number of ChAT immunopositive cells was counted on 10-micron coronal tissue sections. Bilateral counts were performed along the rostrocaudal axis of the lumbar, thoracic, and cervical segments. Cells located in laminae 8 and 9 (ventral horn) of the spinal cord that exhibited a fluorescent ChAT signal were considered motor neurons. Approximately 8 to 10 different levels of each spinal cord segment were counted to generate the overall average number of motor neuron counts per spinal cord segment for each animal. To prevent double counting of the same cell, each section was at least 100 microns apart. Special care was also taken to compare anatomically matched sections between different animals, and cell counts were collected and recorded by a blinded observer.

Myofiber size. For histological analysis of the periphery, the fixed quadriceps, gastrocnemius, and intercostal muscles from the right side of each mouse were processed by paraffin and stained for $\mathrm{H} \& \mathrm{E}$ to determine myofiber cross-section area as reported (16). Approximately 500 nonoverlapping myofibers from each muscle were randomly selected and photographed at $\times 60$ magnification. The cross-section area of each myofiber was then measured using Metamorph (Molecular Devices) to generate the overall average of the myofiber size per muscle for each animal.

NMJ staining. The fixed muscle groups from the left side of each mouse were stored in PBS for NMJ analysis. In toto staining on teased muscle fibers from the quadriceps, gastrocnemius, and intercostal muscles was performed as reported (41). Presynaptic nerve terminals were labeled by overnight incubation at $4{ }^{\circ} \mathrm{C}$ with a rabbit polyclonal antibody against the $150-\mathrm{kDa}$ neurofilament-medium isoform (1:200 dilution; NF-M, Millipore), followed by a biotinylated anti-rabbit secondary antibody (1:200 dilution; Jackson ImmunoResearch). Acetylcholine receptors on the muscle end plates were labeled with Alexa Fluor 555-conjugated $\alpha$-bungarotoxin (Molecular Probes) at 1:5000 for 3 hours at RT. Stained muscle fibers were mounted onto slides, cover-slipped with Vectashield, and viewed under epifluorescence. For NMJ quantification, a minimum of 100 NMJs from each muscle were randomly selected and assessed under the microscope to determine the number of collapsed NMJ for each muscle group per animal. Confocal images were captured using a Zeiss LSM 510-META microscope.

Behavioral tests. In the righting reflex, each mouse was placed in a supine position and the time taken for the mouse to reposition itself onto all 4 paws was measured. The procedure was repeated 3 times for each animal, and the average of the 3 scores was designated the righting score. If the mouse did not respond within 60 seconds, the test was terminated. In the negative-geotaxis, each mouse was placed on a $45^{\circ}$ platform facing downward. The test was deemed a success if the mouse turned $180^{\circ}$ to the "head up" position. Each mouse was given 3 attempts to complete the task in 180 seconds or less. In the grip strength, the fore- 
limbs and hind limbs were placed together on a wire grid and the mouse gently dragged horizontally along the mesh. Resistance was recorded in grams by a force transducer. In the hind-limb splay test, each mouse was suspended by its tail for 5 seconds and the resulting splay was scored based on an arbitrary system. A healthy splay of both hind limbs similar to that observed in WT mice was given a score of 4 . An acute splay angle or "weak splay" of both hind limbs received a score of 3 . A single leg splay was assigned a score of 2 . A mouse that exhibited no splay was given a score of 1 . Finally, a score of 0 occurred when the pup pulled both hind limbs together, effectively crossing one over the other.

Statistics. The behavioral tests, the number of motor neurons, the crosssection myofiber areas, and the NMJ were analyzed with 1-way ANOVA and Bonferroni's multiple post hoc comparisons and with unpaired 2-tailed Student's $t$ tests. The Kaplan-Meier survival curve was analyzed with the log-rank test equivalent to the Mantel-Haenszel test. All statistical analyses were performed with GraphPad Prism v4.0 (GraphPad Software). Values of $P<0.05$ were considered significant.

1. Lefebvre S, et al. Identification and characterization of a spinal muscular atrophy-determining gene. Cell. 1995;80(1):155-165.

2. Sumner CJ. Therapeutics development for spinal muscular atrophy. NeuroRx. 2006;3(2):235-245.

3. Samaha FJ, et al. Pulmonary function in spinal muscular atrophy. J Child Neurol. 1994;9(3):326-329.

4. Iannaccone ST. Modern management of spinal muscular atrophy. J Child Neurol. 2007;22(8):974-978.

5. Monani UR, et al. A single nucleotide difference that alters splicing patterns distinguishes the SMA gene SMN1 from the copy gene SMN2. Hum Mol Genet. 1999;8(7):1177-1183.

6. Lorson CL, Hahnen E, Androphy EJ, Wirth B. A single nucleotide in the SMN gene regulates splicing and is responsible for spinal muscular atrophy. Proc Natl Acad Sci US A. 1999;96(11):6307-6311.

7. Campbell L, Potter A, Ignatius J, Dubowitz V, Davies K. Genomic variation and gene conversion in spinal muscular atrophy: implications for disease process and clinical phenotype. Am J Hum Genet. 1997;61(1):40-50.

8. Lefebvre $\mathrm{S}$, et al. Correlation between severity and SMN protein level in spinal muscular atrophy. Nat Genet. 1997;16(3):265-269.

9. Baron-Delage S, Abadie A, Echaniz-Laguna A, Melki J, Beretta L. Interferons and IRF-1 induce expression of the survival motor neuron (SMN) genes. Mol Med. 2000;6(11):957-968.

10. Brichta L, et al. Valproic acid increases the SMN2 protein level: a well-known drug as a potential therapy for spinal muscular atrophy. Hum Mol Genet. 2003;12(19):2481-2489.

11. Sumner CJ, et al. Valproic acid increases SMN levels in spinal muscular atrophy patient cells. Ann Neurol. 2003;54(5):647-654.

12. Andreassi C, et al. Aclarubicin treatment restores SMN levels to cells derived from type I spinal muscular atrophy patients. Hum Mol Genet. 2001;10(24):2841-2849.

13. Grzeschik SM, Ganta M, Prior TW, Heavlin WD, Wang CH. Hydroxyurea enhances SMN2 gene expression in spinal muscular atrophy cells. Ann Neurol. 2005;58(2):194-202.

14. Hahnen E, et al. In vitro and ex vivo evaluation of second-generation histone deacetylase inhibitors for the treatment of spinal muscular atrophy. J Neurochem. 2006;98(1):193-202.

15. Riessland M, Brichta L, Hahnen E, Wirth B. The benzamide M344, a novel histone deacetylase inhibitor, significantly increases SMN2 RNA/protein levels in spinal muscular atrophy cells. Hum Genet. 2006;120(1):101-110.

16. Avila AM, et al. Trichostatin A increases SMN expression and survival in a mouse model

\section{Acknowledgments}

The authors thank Tatyana Taksir and Kuma Misra (pathology); Antonius Song, Bradley Hodges, Denise Woodcock, Shelley Nass, Matthew DeRiso, and Michael Phipps (molecular biology); Donavon Wagner, Jeff Schoonmaker, Adam Underwood, Teresa Cunio, and Leah Curtin (comparative medicine); Douglas Matthews (confocal); and Robert Thomas (medical illustration) from Genzyme Corporation. In addition, we would like to acknowledge the SMA Foundation for helping us acquire the SMA mouse model.

Received for publication October 29, 2009, and accepted in revised form January 13, 2010.

Address correspondence to: Marco A. Passini, Genzyme Corporation, 49 New York Avenue, Room 2410, Framingham, MA 01701. Phone: 508.270.2033; Fax: 508.271.4776; E-mail: marco.passini@ genzyme.com. of spinal muscular atrophy. $J$ Clin Invest. 2007;117(3):659-671.

17. Andreassi $\mathrm{C}$, et al. Phenylbutyrate increases SMN expression in vitro: relevance for treatment of spinal muscular atrophy. Eur J Hum Genet. 2004;12(1):59-65.

18. Chang JG, et al. Treatment of spinal muscular atrophy by sodium butyrate. Proc Natl Acad Sci U S A. 2001;98(17):9808-9813.

19. Zhang ML, Lorson CL, Androphy EJ, Zhou J. An in vivo reporter system for measuring increased inclusion of exon 7 in SMN2 mRNA: potential therapy of SMA. Gene Ther. 2001;8(20):1532-1538.

20. Angelozzi C, et al. Salbutamol increases SMN mRNA and protein levels in spinal muscular atrophy cells. J Med Genet. 2008;45(1):29-31.

21. Sakla MS, Lorson CL. Induction of full-length survival motor neuron by polyphenol botanical compounds. Hum Genet. 2008;122(6):635-643.

22. Yuo CY, Lin HH, Chang YS, Yang WK, Chang JG. 5-(N-ethyl-N-isopropyl)-amiloride enhances SMN2 exon 7 inclusion and protein expression in spinal muscular atrophy cells. Ann. Neurol. 2008;63(1):26-34.

23. Singh NK, Singh NN, Androphy EJ, Singh RN. Splicing of a critical exon of human Survival Motor Neuron is regulated by a unique silencer element located in the last intron. Mol Cell Biol. 2006;26(4):1333-1346.

24. Hua Y, Vickers TA, Okunola HL, Bennett CF, Krainer AR. Antisense masking of an hnRNP A1/A2 intronic splicing silencer corrects SMN2 splicing in transgenic mice. Am J Hum Genet. 2008;82(4):834-848.

25. Hua Y, Vickers TA, Baker BF, Bennett CF, Krainer AR. Enhancement of SMN2 exon 7 inclusion by antisense oligonucleotides targeting the exon. PLOS Biol. 2007;5:e73.

26. Coady TH, Shababi M, Tullis GE, Lorson CL. Restoration of SMN function: delivery of a trans-splicing RNA re-directs SMN2 pre-mRNA splicing. Mol Ther. 2007;15(8):1471-1478.

27. Coady TH, Baughan TD, Shababi M, Passini MA, Lorson CL. Development of a single vector system that enhances trans-splicing of SMN2 transcripts. PLoS ONE. 2008;3:e3468.

28. Oskoui M, Kaufmann P. Spinal muscular atrophy. Neurotherapentics. 2008;5(4):499-506.

29. Schmid A, DiDonato CJ. Animal models of spinal muscular atrophy. J Child Neurol. 2007;22(8):1004-1012.

30. Le TT, et al. SMN $\Delta 7$, the major product of the centromeric survival motor neuron (SMN2) gene, extends survival in mice with spinal muscular atrophy and associates with full-length SMN. Hum Mol Genet. 2005;14(6):845-857.
31. Narver HL, et al. Sustained improvement of spinal muscular atrophy mice treated with trichostatin A plus nutrition. Ann Neurol. 2008;64(4):465-470.

32. Azzouz M, et al. Lentivector-mediated SMN replacement in a mouse model of spinal muscular atrophy. J Clin Invest. 2004;114(12):1726-1731.

33. Corti $S$, et al. Neural stem cell transplantation can ameliorate the phenotype of a mouse model of spinal muscular atrophy. J Clin Invest. 2008;118(10):3316-3330.

34. McCarthy DM. Self-complementary AAV vectors; advances and applications. Mol Ther. 2008;16(10):1648-1656.

35. Butchbach ME, Edwards JD, Burghes AH. Abnormal motor phenotype in the SMN $\Delta 7$ mouse model of spinal muscular atrophy. Neurobiol Dis. 2007;27(2):207-219.

36. El-Khodor BF, et al. Identification of a battery of tests for drug candidate evaluation in the SMN $\Delta 7$ neonatal model of spinal muscular atrophy. Exp Neurol. 2008;212(1):29-43.

37. Gavrilina TO, et al. Neuronal SMN expression corrects spinal muscular atrophy in severe SMA mice while muscle-specific SMN expression has no phenotypic effect. Hum Mol Genet. 2008;17(8):1063-1075.

38. Murray LM, et al. Selective vulnerability of motor neurons and dissociation of pre- and post-synaptic pathology at the neuromuscular junction in mouse models of spinal muscular atrophy. Hum Mol Genet. 2008;17(7):949-962.

39. Cifuentes-Diaz C, et al. Neurofilament accumulation at the motor endplate and lack of axonal sprouting in a spinal muscular atrophy mouse model. Hum Mol Genet. 2002;11(12):1439-1447.

40. Kariya S, et al. Reduced SMN protein impairs maturation of the neuromuscular junction in mouse models of spinal muscular atrophy. Hum Mol Genet. 2008;17(16):2552-2569.

41. Lesbordes JC, et al. Therapeutic benefits of cardiotrophin-1 gene transfer in a mouse model of spinal muscular atrophy. Hum Mol Genet. 2003;12(11):1233-1239.

42. Rossoll W, et al. SMN, the spinal muscular atrophy-determining gene product, modulates axon growth and localization of B-actin in mRNA in growth cones of motorneurons. J Cell Biol. 2003;163(4):801-812.

43. Burghes AH, Beattie CE. Spinal muscular atrophy: why do low levels of survival motor neuron protein make motor neurons sick? Nat Rev Neurosci. 2009;10(8):597-609.

44. Gabanella F, et al. Ribonucleoprotein assembly defects correlate with spinal muscular atrophy severity and preferentially affect a subset of spli- 
ceosomal snRNPs. PLoS ONE. 2007;2(9):e921.

45. Rudnik-Schöneborn $S$, et al. Congenital heart disease is a feature of severe infantile spinal muscular atrophy. J Med Genet. 2008;45(10):635-638.

46. Wang $\mathrm{CH}$, et al. Consensus statement for standard of care in spinal muscular atrophy. J Child Neurol. 2007;22(8):1027-1049.

47. Husain T, Passini MA, Parente MK, Fraser NW, Wolfe JH. Long-term AAV vector gene and protein expression in mouse brain from a small pan-cellular promoter is similar to neural cell promoters. Gene Ther. 2009;16(7):927-932.

48. Foust KD, et al. Intravascular AAV9 preferentially targets neonatal neurons and adult astrocytes. Nat Biotechnol. 2009;27(1):59-65.

49. Duque $S$, et al. Intravenous administration of self-complementary AAV9 enables transgene delivery to adult motor neurons. Mol Ther.
2009;17(7):1187-1196.

50. Passini MA, Wolfe JH. Widespread gene delivery and structure-specific patterns of expression in the brain after intraventricular injections of neonatal mice with an adeno-associated virus vector. J Virol. 2001;75(24):12382-12392.

51. Zolotukhin I, et al. Recombinant adeno-associated virus production using novel methods improves infectious titer and yield. Gene Ther. 1999;6(6):973-985. 